\title{
Effect of structured discharge teaching after hysterectomy
}

\section{Kshetrimayum Nalini Devi*, Poonam Sheoran, Jyoti Sarin}

M.M.Institute of nursing, M.M.University, Mullana, Haryana, India

Received: 22 July 2015

Revised: 31 July 2015

Accepted: 14 August 2015

\section{*Correspondence:}

Kshetrimayum Nalini Devi,

E-mail: nalinikshe89@gmail.com

Copyright: () the author(s), publisher and licensee Medip Academy. This is an open-access article distributed under the terms of the Creative Commons Attribution Non-Commercial License, which permits unrestricted non-commercial use, distribution, and reproduction in any medium, provided the original work is properly cited.

\section{ABSTRACT}

Background: Hysterectomy is the most common major surgical procedure underwent by women. And there is no proper discharge planning for women underwent hysterectomy. The aim of the study was to assess and compare the self-care ability score of women underwent hysterectomy after implementation of structured discharge teaching in experimental and comparison group.

Methods: The study was quantitative research approach using non-equivalent control group, post-test only design with 60 women underwent hysterectomy selected by purposive sampling technique from gynae ward of Maharishi Markandeshwar Institute of Medical Science \& Research Hospital, Mullana. The post test data was collected by interview technique as $1^{\text {st }}$ follow up visit of operation.

Results: The mean score of self-care ability in experimental group $(103.63 \pm 4.327)$ was higher than mean score of comparison group $(71.67 \pm 4.737)$. The computed ' $t$ ' value was found to be statistically significant at 0.05 level of significant except personal hygiene and exercise. There was significant association of self-care ability score with days of postoperative stay which was found significant at 0.05 level of significant indicating that self-care ability score was dependent on days of postoperative stay $>12$ days.

Conclusions: The findings revealed that the structured discharge teaching on self-care ability was effective on prevention of complication after hysterectomy. Therefore, it is recommended to develop a proper discharge planning protocol regarding self-care after hysterectomy for prevention of complication.

Keywords: Effectiveness, Discharge planning, Self-care ability, Hysterectomy

\section{INTRODUCTION}

One of the goals of health for all in $21^{\text {st }}$ century is the improvement of quality of life; and to find the best treatments for medical problems. Health in the society is the most important factor for social and economic development and every financial or educational investment in this field should be considered essential. Having a good quality of life has always been a wish for human beings. ${ }^{1}$

The World Health Organization (WHO), 2014, defined health as "a state of complete physical, mental, and social well-being and not merely the absence of disease or infirmity." Health has been recognized as the greatest wealth from times immemorial. In present world it is continuously trying to maintain health at optimum level by adapting and practicing different methods. Any deviation from normal health may necessitate medical care for surgical intervention. ${ }^{1}$

Hysterectomy is the most common major surgical procedure underwent by women. ${ }^{1}$ It is the surgical removal of the uterus. It may also involve removal of the cervix, ovaries, fallopian tubes and other surrounding structures. $^{2}$ 
Study found some of the women reported late medical problems after hysterectomy, viz., backache $(44 ; 62.9 \%)$ vaginal discharge $(3 ; 4.3 \%)$, weakness $(11 ; 15.7 \%)$, pain $(11 ; 15.7 \%)$, weight gain $(3 ; 4.3 \%)$, gas $(7 ; 10 \%)$, incontinence $(8 ; 11.4 \%)$ and difficulty in sitting/walking $(5 ; 7.1 \%)$. In $10(14.3 \%)$ cases, feeling of a sense of incompleteness was reported after removal of uterus. ${ }^{2}$

Women after hysterectomy are more likely to develop depression. Signs of depression may include severe and prolonged feelings of sadness and hopelessness; diminished interest in activities; significant weight loss or gain; insomnia; fatigue; and thoughts of death or suicide. However, women who have their ovaries removed during a hysterectomy may experience vaginal dryness and thinness which can make sexual intercourse uncomfortable. These women may also find they have a loss of libido following a hysterectomy due to the drop in the hormone testosterone. ${ }^{2}$

Severe operative complications were more likely in women with a history of serious illness and among those with a higher number of pregnancies. The highest risk was evident among women with fibroids, but the observed continuous reduction in risk associated with age among these women was not apparent along with dysfunctional uterine bleeding. ${ }^{2}$

Discharge planning is also one of the "basic" hospital functions as outlined in Medicare's conditions of participation from Centres for Medicare \& Medicaid Services. The need to establish an effective discharge planning policy and guideline has been given attention, together with the trend toward starting the discharge planning process upon admission, adopting a multidisciplinary approach, and coordinating for postdischarge care support. ${ }^{2}$

\section{METHODS}

The research was conducted on women underwent hysterectomy as a quasi-experimental design with nonequivalent control group, post-test only design. The study was carried out from December 2014 to February 2015 at Maharishi Markandeshwar Institute of Medical Science \& Research Hospital, Mullana.

The research sample includes a total 60 women underwent hysterectomy - 30 experimental group and 30 comparison were selected with purposive sampling technique. The inclusion criteria were women who underwent hysterectomy and admitted in gyane ward of Maharishi Markandeshwar Institute of Medical Science \& Research Hospital, Mullana and are available at the time of data collection.

The instrument used for data collection were description of sample characteristics: demographic variables with 11 items and clinical variables with 13 items and structured self-care ability questionnaire comprised of 44 items covering the area of physical activities, personal hygiene, diet, exercise, emotional stress, psychological activities and sexual activities. The items were graded in 3 point rating scale: "always-3, "sometimes-2" and "never-1" is at minimum 44 and maximum 132.

Data of demographic variables and clinical variables was collected at the time of admission. The first month of data collection was selected as comparison group while those available during the second month were taken into experimental group to prevent from contamination. Daily care was given to both comparison and experimental group and intervention (teaching) on self-care was given for 5 days to prevent from complication. On first day, teaching on self-care regarding breathing exercise, leg exercise and upper and back exercise was given with the help of flash card. On $2^{\text {nd }}$ day, teaching on self-care regarding mobility, pelvic floor exercise, knee bend, knee rolling, pelvic tilting and abdominal exercise was given with the help of flash card. On $3^{\text {rd }}$ day, teaching on selfcare regarding personal hygiene, wound care, emotional stress, psychological activities and tips to deal with emergency was given with the help of flash card. On $4^{\text {th }}$ day, teaching on self-care regarding do's after hysterectomy i.e. walk, pain management, rest, movement and don'ts after hysterectomy i.e. venture out alone, bend or lift, dismiss recovery medications and sexual activity and don'ts after hysterectomy was given with the help of flash card. On $5^{\text {th }}$ day, teaching on selfcare regarding diet to promote healing and to prevent complication was given with the help of flash card. Telephonic follow up and fixing up of appointment was done and post-test was taken on the day of first follow up after discharge from the hospital for both comparison and experimental group.

\section{Ethical consideration}

Ethical approval was obtained from the institutional Ethical Committee of Maharishi Markandeshwar University, Mullana, Ambala, Haryana. Formal administrative permission was obtained from Medical superintendent of Hospital. Written consent was obtained from the study subjects' regarding their willingness to participate in the research project. The purpose for carrying out the research project was explained to the subjects and assurance of confidentiality.

\section{Data analysis}

Data were analysing by using SPSS software. For evaluation of the data, percentage, arithmetic mean and standard deviation, chi-square, t-test in independent groups and ANNOVA's and t test for association.

\section{RESULTS}

Altogether 60 women of experimental and comparison group were homogenous comparable in terms of demographic variables and clinical variables except 
religion. The mean percentage of self-care ability test score of experimental group 78.5 \pm 4.327 was higher than mean percentage of comparison group $54.3 \pm 4.737$. The computed ' $t$ ' value were found statistically significant at 0.5 level of significance. Thus, the mean difference between comparison and experimental group was true difference not by chance indicating teaching was effective in enhancing self-care ability score of women after hysterectomy except personal hygiene and exercise. There was no association of self-care ability of women underwent hysterectomy with demographic variables and clinical variables which were found statistically not significant at 0.05 level except days of post-operative stays for experimental group which was found statistically significant at 0.05 level indicating that selfcare ability score was dependent on days of postoperative stay which means $>12$ days the patient stays at hospital under supervision more self-care ability is developed.

Data presented in Table 1 indicates that half of the women in comparison group (53.3\%) and in experimental group $(56.7 \%)$ were in the age of $31-40$ years. Majority of the women in comparison group (93.3\%) and most of the women in experimental group (70\%) belonged to Hindu religion. Half of the women in comparison group (53.3\%) were from the nuclear family whereas half of the women in experimental group $(53.3 \%)$ were from the joint family and majority of the women in comparison group (93.3\%) and experimental group $(93.3 \%)$ were supported by the family member. All the women in comparison group $(100 \%)$ and experimental group (100\%) were married. Most of the women in comparison group $(60 \%)$ and in experimental group $(53.3 \%)$ were non-literate. All of the women both in comparison group (100\%) and experimental group (100\%) were homemakers. Nearly half of the women in comparison group $(46.7 \%)$ and most of the women in experimental group $(60 \%)$ had monthly income of Rs. 8000-9000/-.Half of the women in comparison group $(50 \%)$ and one third of the women in experimental group (33.3\%) had 2 living children and one third of the women in comparison group $(23.3 \%)$ and experimental group $(16.75 \%)$ had one abortion. One third of the women in comparison group (36.7\%) and in experimental group $(33.3 \%)$ underwent vaginal hysterectomy and most of the women in comparison group (63.3\%) and in experimental group (66.7\%) underwent abdominal hysterectomy.

Chi square between experimental and comparison group were homogenous and comparable in terms of demographic variables except religion.

Data presented in Table 2 indicates that nearly half of the women in comparison group $(40 \%)$ were in blood group B +ve and in experimental group (40\%) were in blood group of A +ve. All the women in experiment (100\%) and comparison group (100\%) were range of $2-7 \mathrm{~min} / \mathrm{sec}$ of bleeding time, $4-10 \mathrm{~min} / \mathrm{sec}$ of clotting time and 5-4.5 lacs $/ \mathrm{mm}^{3}$ of platelet count respectively. Most of the women in comparison group (70\%) and in experimental group $(73.3 \%)$ had stayed in hospital 1-3 days before hysterectomy. Majority of the women in comparison group $(63.3 \%)$ and in experimental group $(86.7 \%)$ were range of $7 \mathrm{gm} \%-11 \mathrm{gm} \%$ of haemoglobin. Most of the women in comparison group $(83.3 \%)$ and in experimental group $(73.3 \%)$ did not received blood transfusion. Majority of the women in comparison group (80\%) and in experimental group (93.3\%) had stayed 6-8 days after hysterectomy, nearly half of the women in comparison group $(46.7 \%)$ and majority of the women in experimental group $(76.7 \%)$ had stayed $7-11$ days in the hospital. One third of the women in comparison group $(40 \%)$ and in experimental group (40\%) had discharge on $7^{\text {th }}$ day after hysterectomy. One third of the women in comparison group $(30 \%)$ had come on $9^{\text {th }}$ day of follow up and in experimental group $(26.7 \%)$ had come on $8^{\text {th }}$ day of follow up. All the women in comparison group $(100 \%)$ and in experimental group $(100 \%)$ did not have any complaints of complication after operation.

Chi square between experimental and comparison group were homogenous and comparable in terms of clinical variable.

Data presented in Table 3 represents the self-care ability for both comparison group and experimental group. The data indicated that after implementation of structured discharge planning, mean percentage of self-care ability test score of experimental group $78.5 \pm 4.327$ was higher than mean percentage of comparison group $54.3 \pm 4.737$.

Data presented in Table 4 represents the mean score of comparison group were physical activities (17.4), diet (21.36), emotional stress (8.86), psychological activities (6.26) and sexual activities (1.00) whereas in experimental group were physical activities (32.8), diet (40.83), emotional stress (16.30), psychological activities (9.53) and sexual activities (2.67) respectively. The computed ' $\mathrm{t}$ ' value were found statistically significant at 0.5 level of significance. Thus, the mean difference between comparison and experimental group was true difference not by chance indicating structured discharge planning was effective in enhancing self-care ability score of women after hysterectomy.

The mean score of personal hygiene (9.86) and exercise (5.90) in comparison group whereas the mean score of personal hygiene (18.5) and (10.76) in experimental group respectively. The computed ' $t$ ' value were found statistically non-significant at 0.5 level of significance indicating that the discharge was not effective in the area of personal hygiene and exercise. 
Table 1: Percentage and frequency distribution of women underwent hysterectomy according to selected demographic variables.

\begin{tabular}{|c|c|c|c|c|c|}
\hline Demographic Variables & $\begin{array}{l}\text { Comparison group } \\
\mathbf{f}(\%) \\
(\mathrm{n}=\mathbf{3 0})\end{array}$ & $\begin{array}{l}\text { Experimental group } \\
\mathrm{f}(\%) \\
(\mathrm{n}=\mathbf{3 0})\end{array}$ & Chi/Yates & df & $\begin{array}{l}\text { P- } \\
\text { Value }\end{array}$ \\
\hline \multicolumn{6}{|l|}{ 1.Age } \\
\hline $1.1 \leq 30$ years & $2(6.7 \%)$ & $4(13.3 \%)$ & 14.696 & 3 & $0.327^{\mathrm{NS}}$ \\
\hline $1.231-40$ years & $16(53.3 \%)$ & $17(56.7 \%)$ & & & \\
\hline $1.341-50$ years & $9(30 \%)$ & $7(23.3 \%)$ & & & \\
\hline $1.451-60$ years & $3(10 \%)$ & $2(6.7 \%)$ & & & \\
\hline \multicolumn{6}{|l|}{ 2. Religion } \\
\hline $2.1 \mathrm{Hindu}$ & $28(93.3 \%)$ & $21(70 \%)$ & 12.00 & 2 & $0.002^{*}$ \\
\hline $2.2 \mathrm{Sikh}$ & $2(6.7 \%)$ & 0 & & & \\
\hline 2.3 Muslim & 0 & $9(30 \%)$ & & & \\
\hline \multicolumn{6}{|l|}{ 3. Types of family } \\
\hline 3.1 Nuclear & $16(53.3 \%)$ & $14(46.7 \%)$ & 0.267 & 1 & $0.606^{\mathrm{NS}}$ \\
\hline 3.2 Joint & $14(46.7 \%)$ & $16(53.3 \%)$ & & & \\
\hline \multicolumn{6}{|c|}{$\begin{array}{l}\text { Whether there is any social support for } \\
\text { the women at home? }\end{array}$} \\
\hline Yes & $28(93.3 \%)$ & $28(93.3 \%)$ & 0.00 & 1 & $1.000^{\mathrm{NS}}$ \\
\hline No & $2(6.7 \%)$ & $2(6.7 \%)$ & & & \\
\hline \multicolumn{6}{|l|}{ 4. Marital status } \\
\hline 4.1 Married & $30(100 \%)$ & $30(100 \%)$ & NA & & \\
\hline \multicolumn{6}{|l|}{ 5. Educational status } \\
\hline 5.1 Non-literate & $18(60 \%)$ & $16(53.3 \%)$ & 0.618 & 2 & $0.734^{\mathrm{NS}}$ \\
\hline 5.2 Primary education & $9(30 \%)$ & $9(30 \%)$ & & & \\
\hline 5.3 Secondary education & $3(10 \%)$ & $5(16.7 \%)$ & & & \\
\hline \multicolumn{6}{|l|}{ 6. Occupation } \\
\hline 6.1 Homemaker & $30(100 \%)$ & $30(100 \%)$ & NA & & \\
\hline \multicolumn{6}{|l|}{ 7. Monthly income in rupees } \\
\hline $7.1 \leq 8000$ & $11(36.7 \%)$ & $7(23.3 \%)$ & 18.92 & 3 & $0.333^{\mathrm{NS}}$ \\
\hline $7.28001-9000$ & $14(46.7 \%)$ & $18(60 \%)$ & & & \\
\hline 7.390001 and above & $5(16.7 \%)$ & $5(16.7 \%)$ & & & \\
\hline \multicolumn{6}{|l|}{ 8. Parity } \\
\hline \multicolumn{6}{|l|}{ a) No. of living children } \\
\hline 8.1a. 10 & 0 & $3(10 \%)$ & 5.440 & 4 & $0.245^{\mathrm{NS}}$ \\
\hline 8.2a. 22 & $15(50 \%)$ & $10(33.3 \%)$ & & & \\
\hline 8.3a. 33 & $9(30 \%)$ & $6(20 \%)$ & & & \\
\hline 8.4a. 44 & $6(20 \%)$ & $5(16.7 \%)$ & & & \\
\hline 8.5 a. 55 & 0 & $5(16.7 \%)$ & & & \\
\hline $8.6 a .66$ & 0 & $1(3.3 \%)$ & & & \\
\hline \multicolumn{6}{|l|}{ b) No. Of abortion } \\
\hline $8.1 \mathrm{~b} .10$ & $23(73.3 \%)$ & $25(82.25 \%)$ & 1.826 & 1 & $0.177^{\mathrm{NS}}$ \\
\hline $8.2 \mathrm{~b} .21$ & $7(23.3 \%)$ & $5(16.7 \%)$ & & & \\
\hline \multicolumn{6}{|l|}{ 9. Types of operation } \\
\hline 9.1 Vaginal hysterectomy & $11(36.7 \%)$ & $10(33.3 \%)$ & 0.73 & 1 & $0.787^{\mathrm{NS}}$ \\
\hline 9.2 Abdominal hysterectomy & $19(63.3 \%)$ & $20(66.7 \%)$ & & & \\
\hline \multicolumn{6}{|l|}{ 10. Indication of operation } \\
\hline 10.1 Fibroid uterus & $15(50 \%)$ & $16(53.3 \%)$ & 4.389 & 4 & $0.356^{\mathrm{NS}}$ \\
\hline 10.2 UV prolapsed & $11(36.7 \%)$ & $10(33.3 \%)$ & & & \\
\hline $10.3 \mathrm{AUB}$ & $4(13.3 \%)$ & $4(13.3 \%)$ & & & \\
\hline \multicolumn{6}{|l|}{ Co-morbid disease } \\
\hline Yes & $7(23.3 \%)$ & $9(30 \%)$ & 0.009 & 1 & $0.925^{\mathrm{NS}}$ \\
\hline No & $23(76.7 \%)$ & $21(70 \%)$ & & & \\
\hline
\end{tabular}


Table 2: Percentage and frequency distribution of women underwent hysterectomy according to preoperative \& postoperative clinical variables.

\begin{tabular}{|c|c|c|c|c|c|}
\hline Clinical Variables & $\begin{array}{l}\text { Comparison group } \\
\mathbf{f}(\%) \\
(\mathbf{n}=\mathbf{3 0})\end{array}$ & $\begin{array}{l}\text { Experimental group } \\
\mathrm{f}(\%) \\
(\mathrm{n}=\mathbf{3 0})\end{array}$ & Chi/ Yates & df & $P$ value \\
\hline \multicolumn{6}{|l|}{ Preoperative } \\
\hline \multicolumn{6}{|l|}{ 1. Blood group } \\
\hline $1.1 \mathrm{~A}+\mathrm{ve}$ & $9(30 \%)$ & $12(40 \%)$ & 1.396 & 3 & $0.706^{\mathrm{NS}}$ \\
\hline $1.2 \mathrm{~B}+\mathrm{ve}$ & $12(40 \%)$ & $7(23.3 \%)$ & & & \\
\hline $1.3 \mathrm{O}+\mathrm{ve}$ & $5(16.7 \%)$ & $4(13.3 \%)$ & & & \\
\hline $1.4 \mathrm{AB}+\mathrm{ve}$ & $4(13.3 \%)$ & $7(23.3 \%)$ & & & \\
\hline \multicolumn{6}{|l|}{ 2. Bleeding time } \\
\hline $2.1<2 \mathrm{~min} / \mathrm{sec}$ & 0 & 0 & NA & & \\
\hline $2.22-7 \mathrm{~min} / \mathrm{sec}$ & $30(100 \%)$ & $30(100 \%)$ & & & \\
\hline $2.3>7 \mathrm{~min} / \mathrm{sec}$ & 0 & 0 & & & \\
\hline \multicolumn{6}{|l|}{ 3. Clotting time } \\
\hline $3.1<4 \mathrm{~min} / \mathrm{sec}$ & 0 & 0 & NA & & \\
\hline $3.24-10 \mathrm{~min} / \mathrm{sec}$ & $30(100 \%)$ & $30(100 \%)$ & & & \\
\hline $3.3>10 \mathrm{~min} / \mathrm{sec}$ & 0 & 0 & & & \\
\hline \multicolumn{6}{|l|}{ 4. Platelet count } \\
\hline $4.1<1$. lacs $/ \mathrm{mm}^{3}$ & 0 & 0 & NA & & \\
\hline $4.21 .5-4.5 \mathrm{lacs} / \mathrm{mm}^{3}$ & $30(100 \%)$ & $30(100 \%)$ & & & \\
\hline $4.3>4.5 \mathrm{lacs} / \mathrm{mm}^{3}$ & 0 & 0 & & & \\
\hline \multicolumn{6}{|c|}{ 5. Days of pre-operative stay } \\
\hline $5.11-3$ & $21(70 \%)$ & $22(73.3 \%)$ & 2.953 & 2 & $0.228^{\mathrm{NS}}$ \\
\hline $5.24-6$ & $5(16.7 \%)$ & $8(26.7 \%)$ & & & \\
\hline $5.37-9$ & $4(13.3 \%)$ & 0 & & & \\
\hline \multicolumn{6}{|l|}{ Postoperative } \\
\hline \multicolumn{6}{|l|}{ 1) Haemoglobin } \\
\hline $1.1<3 \mathrm{gm} \%$ & 0 & 0 & 4.883 & 3 & $0.087^{\mathrm{NS}}$ \\
\hline $1.23 \mathrm{gm} \%-6.9 \mathrm{gm} \%$ & $1(3.3 \%)$ & $2(6.7 \%)$ & & & \\
\hline $1.37 \mathrm{gm} \%-11 \mathrm{gm} \%$ & $19(63.3 \%)$ & $26(86.7 \%)$ & & & \\
\hline $1.4>11 \mathrm{gm} \%$ & $10(33.3 \%)$ & $2(6.7 \%)$ & & & \\
\hline \multicolumn{6}{|l|}{ 2) Blood transfusion } \\
\hline 2.10 unit & $25(83.3 \%)$ & $22(73.3 \%)$ & 0.530 & 3 & $0.912^{\mathrm{NS}}$ \\
\hline 2.21 unit & $3(10 \%)$ & $6(20 \%)$ & & & \\
\hline 2.32 unit & $1(3.3 \%)$ & $2(6.7 \%)$ & & & \\
\hline 2.43 unit & $1(3.3 \%)$ & 0 & & & \\
\hline $2.5>4$ unit & 0 & 0 & & & \\
\hline \multicolumn{6}{|l|}{ 3) TLC } \\
\hline $3.1<5,000$ cumm & 0 & 0 & NA & & \\
\hline $3.25,000-11,000$ cumm & $30(100 \%)$ & $30(100 \%)$ & & & \\
\hline $3.3>11,000 \mathrm{cumm}$ & 0 & 0 & & & \\
\hline \multicolumn{6}{|c|}{ 4) Days of post-operative stay } \\
\hline $4.16-8$ & $24(80 \%)$ & $28(93.3 \%)$ & 4.84 & 2 & $0.089^{\mathrm{NS}}$ \\
\hline $4.29-11$ & $6(20 \%)$ & 0 & & & \\
\hline $4.312-14$ & 0 & $2(6.7 \%)$ & & & \\
\hline \multicolumn{6}{|c|}{ 5) Duration of hospital stay } \\
\hline $5.17-11$ & $14(46.7 \%)$ & $23(76.7 \%)$ & 3.919 & 2 & $0.141^{\mathrm{NS}}$ \\
\hline $5.212-16$ & $12(40 \%)$ & $6(20 \%)$ & & & \\
\hline $5.317-21$ & $4(13.3 \%)$ & $1(3.3 \%)$ & & & \\
\hline \multicolumn{6}{|l|}{ 6) Day of discharge } \\
\hline $6.16^{\text {th }}$ day of operation & $7(23.3 \%)$ & $9(30 \%)$ & 3.327 & 4 & $0.505^{\mathrm{NS}}$ \\
\hline $6.27^{\text {th }}$ day of operation & $12(40 \%)$ & $12(40 \%)$ & & & \\
\hline
\end{tabular}




\begin{tabular}{|lllll|}
\hline $6.38^{\text {th }}$ day of operation & $6(20 \%)$ & $7(23.3 \%)$ & & \\
\hline $6.49^{\text {th }}$ day of operation & $2(6.7 \%)$ & $2(6.7 \%)$ & & \\
\hline $6.510^{\text {th }}$ day of operation & $3(10 \%)$ & 0 & & \\
\hline 7$)$ Days of follow up after discharge & & & & \\
\hline $7.17^{\text {th }}$ day & $5(16.7 \%)$ & $5(16.7 \%)$ & & \\
\hline $7.28^{\text {th }}$ day & $1(3.3 \%)$ & $8(26.7 \%)$ & & \\
\hline $7.39^{\text {th }}$ day & $9(30 \%)$ & $6(20 \%)$ & & \\
\hline $7.410^{\text {th }}$ day & $6(20 \%)$ & $4(13.3 \%)$ & \\
\hline $7.511^{\text {th }}$ day & $4(13.3 \%)$ & $5(16.7 \%)$ & \\
\hline $7.612^{\text {th }}$ day & $4(13.3 \%)$ & $2(6.7 \%)$ & & \\
\hline $7.713^{\text {th }}$ day & $1(3.3 \%)$ & & & \\
\hline 8$)$ Any post-operative complication & & & & \\
\hline 8.1 Yes & 0 & 0 & & \\
\hline $8.2 \mathrm{No}$ & $30(100 \%)$ & $30(100 \%)$ & \\
\hline
\end{tabular}

Table 3: Range, mean, median, standard deviation and mean percentage of self-care ability of women underwent hysterectomy in comparison and experimental group.

\begin{tabular}{|lllllll|} 
& Group & Range & Mean & Mean \% & Median & SD \\
\hline $\begin{array}{l}\text { Self-care } \\
\text { Ability }\end{array}$ & Comparison & $63-79$ & 71.68 & $54.3 \%$ & 72.5 & 4.737 \\
\cline { 2 - 6 } & Experimental & $96-113$ & 103.63 & $78.5 \%$ & 104 & 4.327 \\
\hline
\end{tabular}

Table 4: Area wise mean, mean difference, standard deviation of difference, standard error of mean difference and ' $t$ ' value of self care ability score of women underwent hysterectomy in comparison group and experimental group.

\begin{tabular}{|c|c|c|c|c|c|c|c|}
\hline \multicolumn{2}{|c|}{ Self-Care Ability } & $\begin{array}{l}\text { Mean } \\
\text { Score }\end{array}$ & $\begin{array}{l}\text { Mean } \\
\text { Difference }\end{array}$ & SDd & SEmd & t Value & P value \\
\hline \multirow{2}{*}{$\begin{array}{l}\text { Physical } \\
\text { Activities }\end{array}$} & Comparison & 17.4 & 15.43 & 2.51 & 1.15 & 13.416 & $0.02 *$ \\
\hline & Experimental & 32.8 & & & & & \\
\hline \multirow{2}{*}{$\begin{array}{l}\text { Personal } \\
\text { Hygiene }\end{array}$} & Comparison & 9.86 & 8.63 & 1.56 & 0.74 & 11.587 & $0.12^{\mathrm{NS}}$ \\
\hline & Experimental & 18.5 & & & & & \\
\hline \multirow[t]{2}{*}{ Diet } & Comparison & 21.36 & 19.46 & 1.76 & 0.74 & 26.177 & $0.00^{*}$ \\
\hline & Experimental & 40.83 & & & & & \\
\hline \multirow{2}{*}{ Exercise } & Comparison & 5.90 & 4.86 & 0.31 & 15.296 & 15.296 & $0.61^{\mathrm{NS}}$ \\
\hline & Experimental & 10.76 & & & & & \\
\hline \multirow{2}{*}{$\begin{array}{l}\text { Emotional } \\
\text { Stress }\end{array}$} & Comparison & 8.86 & 43 & 1.13 & 0.54 & 13.604 & $0.00^{*}$ \\
\hline & Experimental & 16.30 & & & & & \\
\hline \multirow{2}{*}{$\begin{array}{l}\text { Psychosocial } \\
\text { Activities }\end{array}$} & Comparison & 6.26 & 3.26 & 0.71 & 3.70 & 8.820 & $0.01^{*}$ \\
\hline & Experimental & 9.53 & & & & & \\
\hline \multirow{2}{*}{$\begin{array}{l}\text { Sexual } \\
\text { Activities }\end{array}$} & Comparison & 1.00 & 1.67 & 0.479 & 0.87 & 19.039 & $0.00^{*}$ \\
\hline & Experimental & 2.67 & & & & & \\
\hline
\end{tabular}

The table 5 depicted that the association of self-care ability of women underwent hysterectomy with selected demographic variables i.e. Age $(F=1.820, p=0.168)$, religion $(\mathrm{t}=0.518, \mathrm{p}=0.608)$, types of family $(\mathrm{t}=0.744$, $\mathrm{p}=0.463)$, social support $(\mathrm{t}=0.122, \mathrm{p}=0.904)$, educational status $(\mathrm{F}=0.172, \mathrm{p}=0.843)$, monthly income in rupees $(\mathrm{F}=0.471, \mathrm{p}=0.630)$, parity- number of living children $(\mathrm{F}=0.250, \mathrm{p}=0.936)$ and number of abortion $(\mathrm{t}=0.241, \mathrm{p}=0.811)$, types of operation $(\mathrm{t}=$ $1.592, \mathrm{p}=0.123)$, indication of operation $(\mathrm{F}=1.572, \mathrm{p}=$ $0.226)$ with any co morbid disease $(\mathrm{t}=0.666, \mathrm{p}=0.511)$ of experimental group was found not significant at 0.05 level of significant. 
Thus it can be inferred that there was no association of self-care ability with age, religion, types of family, marital status, educational status, occupation, monthly income in rupees, parity, types of operation, indication of operation with any co morbid disease in experimental group.

Table 5: ANOVAs and t test showing association of self care ability of women underwent hysterectomy with selected demographic variables in comparison group.

\begin{tabular}{|c|c|c|c|c|}
\hline Demographic Variables & Mean & $\mathrm{f} / \mathrm{t}$ test & df & P-value \\
\hline \multicolumn{5}{|l|}{ 1) Age } \\
\hline $1.1 \leq 30$ years & 75.50 & 2.588 & $3 / 26$ & $0.75^{\mathrm{NS}}$ \\
\hline $1.231-40$ years & 71.25 & & & \\
\hline $1.341-50$ years & 68.77 & & & \\
\hline $1.451-60$ years & 77.00 & & & \\
\hline \multicolumn{5}{|l|}{ 2) Religion } \\
\hline 2.1 Hindu & 71.64 & 0.101 & 28 & $0.920^{\mathrm{NS}}$ \\
\hline 2.2 Sikh & 72.00 & & & \\
\hline \multicolumn{5}{|l|}{ 3) Types of family } \\
\hline 3.1 Nuclear & 72.31 & 0.793 & 28 & $0.343^{\mathrm{NS}}$ \\
\hline 3.2 Joint & 70.92 & & & \\
\hline \multicolumn{5}{|c|}{ Whether there is any social support for the women at home? } \\
\hline Yes & 69.00 & 0.819 & 28 & $0.419^{\mathrm{NS}}$ \\
\hline No & 71.85 & & & \\
\hline \multicolumn{5}{|l|}{ 4) Educational status } \\
\hline 4.1Non-literate & 71.61 & 1.843 & $2 / 27$ & $0.178^{\mathrm{NS}}$ \\
\hline 4.2 Primary education & 73.22 & & & \\
\hline 4.3 Secondary education & 67.33 & & & \\
\hline \multicolumn{5}{|l|}{ 5) Monthly income in rupees } \\
\hline $5.1 \leq 8000$ & 70.36 & 2.448 & $2 / 27$ & $0.105^{\mathrm{NS}}$ \\
\hline $5.28001-9000$ & 73.57 & & & \\
\hline 5.390001 and above & 69.20 & & & \\
\hline \multicolumn{5}{|l|}{ 6) Parity } \\
\hline \multicolumn{5}{|l|}{ a) No. of living children } \\
\hline $6 a .22$ & 70.60 & 1.808 & $2 / 27$ & $0.245^{\mathrm{NS}}$ \\
\hline 6a.3 3 & 74.11 & & & \\
\hline $6 a .44$ & 70.66 & & & \\
\hline \multicolumn{5}{|l|}{ b) No. of abortion } \\
\hline $6 \mathrm{~b} .10$ & 72.43 & 1.658 & 28 & $0.109^{\mathrm{NS}}$ \\
\hline $6 b .21$ & 69.14 & & & \\
\hline \multicolumn{5}{|l|}{ 7) Types of operation } \\
\hline 7.1Vaginal hysterectomy & 73.36 & 1.527 & 28 & $0.138^{\mathrm{NS}}$ \\
\hline 7.2Abdominal hysterectomy & 70.68 & & & \\
\hline \multicolumn{5}{|l|}{ 8) Indication of operation } \\
\hline 8.1Fibroid uterus & 70.86 & 0.699 & $2 / 27$ & $0.506^{\mathrm{NS}}$ \\
\hline 8.2UV prolapsed & 71.90 & & & \\
\hline 8.3AUB & 74.00 & & & \\
\hline \multicolumn{5}{|l|}{ Co-morbid disease } \\
\hline Yes & 72.57 & 0.570 & 28 & $0.573^{\mathrm{NS}}$ \\
\hline No & 71.39 & & & \\
\hline
\end{tabular}

The table 6 depicted that the association of self-care ability of women underwent hysterectomy with selected demographic variables i.e. Age $(\mathrm{F}=1.820, \mathrm{p}=0.168)$, religion $(\mathrm{t}=0.518, \mathrm{p}=0.608)$, types of family $(\mathrm{t}=0.744$, $\mathrm{p}=0.463)$, social support $(\mathrm{t}=0.122, \mathrm{p}=0.904)$, educational status $(\mathrm{F}=0.172, \mathrm{p}=0.843)$, monthly income in rupees $(\mathrm{F}=0.471, \mathrm{p}=0.630)$, parity- number of living children $(\mathrm{F}=0.250, \mathrm{p}=0.936)$ and number of 
abortion $(\mathrm{t}=0.241, \mathrm{p}=0.811)$, types of operation $(\mathrm{t}=$ $1.592, \mathrm{p}=0.123)$, indication of operation $(\mathrm{F}=1.572, \mathrm{p}=$ $0.226)$ with any co morbid disease $(\mathrm{t}=0.666, \mathrm{p}=0.511)$ of experimental group was found not significant at 0.05 level of significant. Thus it can be inferred that there was no association of self-care ability with age, religion, types of family, marital status, educational status, occupation, monthly income in rupees, parity, types of operation, indication of operation with any co morbid disease in experimental group.

Table 6: ANOVAs and t test showing association of self care ability score of women underwent hysterectomy with selected demographic variables in experimental group.

\begin{tabular}{|c|c|c|c|c|}
\hline Demographic Variables & Mean & F/t test & DF & P value \\
\hline \multicolumn{5}{|l|}{ 1) Age } \\
\hline $1.1 \leq 30$ years & 100.75 & 1.820 & $3 / 26$ & $0.168^{\mathrm{NS}}$ \\
\hline $1.231-40$ years & 104.88 & & & \\
\hline $1.341-50$ years & 103.42 & & & \\
\hline $1.451-60$ years & 99.50 & & & \\
\hline \multicolumn{5}{|l|}{ 2) Religion } \\
\hline 2.1 Hindu & 103.90 & 0.518 & 28 & $0.608^{\mathrm{NS}}$ \\
\hline 2.2 Sikh & 103.00 & & & \\
\hline \multicolumn{5}{|l|}{ 3) Types of family } \\
\hline 3.1 Nuclear & 103.00 & 0.744 & 28 & $0.463^{\mathrm{NS}}$ \\
\hline 3.2 Joint & 104.18 & & & \\
\hline \multicolumn{5}{|c|}{ Whether there is any social support for the women at home? } \\
\hline Yes & 104.00 & 0.122 & 28 & $0.904^{\mathrm{NS}}$ \\
\hline No & 103.60 & & & \\
\hline \multicolumn{5}{|l|}{ 4) Educational status } \\
\hline 4.1 Non-literate & 103.18 & 0.172 & $2 / 27$ & $0.843^{\mathrm{NS}}$ \\
\hline 4.2 Primary education & 104.11 & & & \\
\hline 4.3 Secondary education & 104.20 & & & \\
\hline \multicolumn{5}{|l|}{ 5) Monthly income in rupees } \\
\hline $5.1 \leq 8000$ & 102.71 & 0.471 & $2 / 27$ & $0.630^{\mathrm{NS}}$ \\
\hline $5.28001-9000$ & 103.55 & & & \\
\hline 5.390001 and above & 105.20 & & & \\
\hline \multicolumn{5}{|l|}{ 6) Parity } \\
\hline \multicolumn{5}{|l|}{ a) No. Of living children } \\
\hline $6 a .10$ & 103.00 & 0.250 & $5 / 24$ & $0.936^{\mathrm{NS}}$ \\
\hline $6 a .22$ & 103.30 & & & \\
\hline $6 a .33$ & 103.00 & & & \\
\hline $6 a .44$ & 105.60 & & & \\
\hline $6 a .55$ & 103.20 & & & \\
\hline $6 a .66$ & 105.00 & & & \\
\hline \multicolumn{5}{|l|}{ b) No. Of abortion } \\
\hline 6b.10 & 103.72 & 0.241 & 28 & $0.811^{\mathrm{NS}}$ \\
\hline $6 b .21$ & 103.20 & & & \\
\hline \multicolumn{5}{|l|}{ 7) Types of operation } \\
\hline 7.1 Vaginal hysterectomy & 101.90 & 1.592 & 28 & $0.123^{\mathrm{NS}}$ \\
\hline 7.2 Abdominal hysterectomy & 104.50 & & & \\
\hline \multicolumn{5}{|l|}{ 8) Indication of operation } \\
\hline 8.1 Fibroid uterus & 104.41 & 1.572 & $2 / 27$ & $0.226^{\mathrm{NS}}$ \\
\hline 8.2 UV prolapsed & 101.55 & & & \\
\hline 8.3 AUB & 105.00 & & & \\
\hline \multicolumn{5}{|l|}{ Co-morbid disease } \\
\hline Yes & 101.44 & 0.666 & 28 & $0.511^{\mathrm{NS}}$ \\
\hline No & 103.28 & & & \\
\hline
\end{tabular}


Table 7: ANOVAs and $\mathrm{t}$ test showing association of self care ability of women underwent hysterectomy with selected preoperative and postoperative clinical variables in comparison group.

\begin{tabular}{|c|c|c|c|c|}
\hline Clinical Variables & Mean & F/t test & DF & P value \\
\hline \multicolumn{5}{|l|}{ Preoperative } \\
\hline \multicolumn{5}{|l|}{ 1) Blood group } \\
\hline $1.1 \mathrm{~A}+\mathrm{ve}$ & 72.44 & 0.454 & $3 / 26$ & $0.717^{\mathrm{NS}}$ \\
\hline $1.2 \mathrm{~B}+\mathrm{ve}$, & 72.25 & & & \\
\hline $1.3 \mathrm{O}+\mathrm{ve}$ & 69.80 & & & \\
\hline 1.4 AB+ve, & 70.50 & & & \\
\hline \multicolumn{5}{|c|}{ 2) Days of pre-operative stay } \\
\hline $2.11-3$ & 71.14 & 1.155 & $2 / 27$ & $0.330^{\mathrm{NS}}$ \\
\hline $2.24-6$ & 71.20 & & & \\
\hline $2.37-9$ & 75.00 & & & \\
\hline \multicolumn{5}{|l|}{ Postoperative } \\
\hline \multicolumn{5}{|l|}{ 1) Haemoglobin } \\
\hline $1.1<3 \mathrm{gm} \%$ & 72.00 & 1.654 & $2 / 27$ & $0.732^{\mathrm{NS}}$ \\
\hline $1.23 \mathrm{gm} \%-6.9 \mathrm{gm} \%$ & 72.78 & & & \\
\hline $1.37 \mathrm{gm} \%-11 \mathrm{gm} \%$ & 69.50 & & & \\
\hline \multicolumn{5}{|l|}{$1.4>11 \mathrm{gm} \%$} \\
\hline \multicolumn{5}{|l|}{ 2) Blood transfusion } \\
\hline 5.20 unit & 71.36 & 0.259 & $3 / 26$ & $0.854^{\mathrm{NS}}$ \\
\hline 5.31 unit & 74.00 & & & \\
\hline 5.42 unit & 72.00 & & & \\
\hline 5.53 unit & 72.00 & & & \\
\hline \multicolumn{5}{|c|}{ 3) Days of post-operative stay } \\
\hline $3.16-8$ & 71.75 & 0.189 & 28 & $0.851^{\mathrm{NS}}$ \\
\hline $3.29-11$ & 71.33 & & & \\
\hline \multicolumn{5}{|l|}{ 4) Duration of hospital stay } \\
\hline $4.17-11$ & 72.14 & 1.918 & $2 / 27$ & $0.166^{\mathrm{NS}}$ \\
\hline $4.212-16$ & 70.00 & & & \\
\hline $4.317-21$ & 75.00 & & & \\
\hline \multicolumn{5}{|l|}{ 5) Day of discharge } \\
\hline $5.16^{\text {th }}$ day of operation & 74.57 & 2.050 & $4 / 25$ & $0.118^{\mathrm{NS}}$ \\
\hline $5.27^{\text {th }}$ day of operation & 69.75 & & & \\
\hline $5.38^{\text {th }}$ day of operation & 72.16 & & & \\
\hline $5.49^{\text {th }}$ day of operation & 67.50 & & & \\
\hline $5.510^{\text {th }}$ day of operation & 74.33 & & & \\
\hline \multicolumn{5}{|c|}{ 6) Days of follow up after discharge } \\
\hline $6.17^{\text {th }}$ day & 68.20 & 1.604 & $6 / 23$ & $0.592^{\mathrm{NS}}$ \\
\hline $6.28^{\text {th }}$ day & 79.00 & & & \\
\hline $6.39^{\text {th }}$ day & 73.22 & & & \\
\hline $6.410^{\text {th }}$ day & 71.16 & & & \\
\hline $6.511^{\text {th }}$ day & 71.75 & & & \\
\hline $6.612^{\text {th }}$ day & 69.75 & & & \\
\hline $6.713^{\text {th }}$ day & 71.66 & & & \\
\hline
\end{tabular}

The table 7 depicted that there was no association of selfcare ability of women underwent hysterectomy with selected clinical variables i.e. blood group $(\mathrm{F}=0.454, \mathrm{p}=$ 0.717), haemoglobin $(\mathrm{F}=1.654, \mathrm{p}=0.732)$, blood transfusion $(\mathrm{F}=0.259, \mathrm{p}=0.854)$, days of pre-operative stay $(\mathrm{F}=1.155, \mathrm{p}=0.330)$, days of post-operative stay $(\mathrm{t}$ $=0.189, \mathrm{p}=0.851)$, duration of hospital stay $(\mathrm{F}=1.918$, $\mathrm{p}=0.166)$, day of discharge $(\mathrm{F}=2.050, \mathrm{p}=0.118)$ and day of follow up $(\mathrm{F}=1.604, \mathrm{p}=0.191)$ of comparison group were found not significant at 0.05 level of significant indicating that self-care ability score of women underwent hysterectomy were not dependent on age, religion, types of family, social support, marital status, educational status, occupation, monthly income in rupees, parity, types of operation, indication of operation with any co morbid disease. 
The table 8 shows that there was significant association of self-care ability with days of postoperative stay $(\mathrm{t}=2.762, \mathrm{p}=0.010)$ which was found statistically significant at 0.05 level indicating that self-care ability score was dependent on days of postoperative stay which means $>12$ days the patient stays at hospital under supervision more self-care ability is developed. Further, there was no significant association of blood group $(\mathrm{F}=1.367, \mathrm{p}=0.275)$, haemoglobin $(\mathrm{t}=0.459, \mathrm{p}=$
0.305), blood transfusion $(F=0.526, p=0.637)$, days of pre-operative stay $(\mathrm{F}=1.045, \mathrm{p}=0.597)$, duration of hospital stay $(\mathrm{F}=1.729, \mathrm{p}=0.197)$, day of discharge $(\mathrm{F}=$ 2.827, $\mathrm{p}=0.118)$ and day of follow up $(\mathrm{F}=0.435, \mathrm{p}=$ 0.820) of experimental group were not found statistically significant at 0.05 level of significant.

Thus, null hypothesis $\mathrm{H}_{3}$ was partially accepted and research hypothesis $\mathrm{H}_{03}$ was partially rejected.

Table 8: ANOVAs and t test showing association of level of mean gain after implementation of discharge planning on self care ability of women underwent hysterectomy with selected preoperative and postoperative clinical variables in experimental group.

\begin{tabular}{|c|c|c|c|c|}
\hline Clinical Variables & Mean & F/t test & DF & $P$ value \\
\hline \multicolumn{5}{|l|}{ Preoperative } \\
\hline \multicolumn{5}{|l|}{ 1) Blood group } \\
\hline $1.1 \mathrm{~A}+\mathrm{ve}$ & 105.16 & 1.367 & $3 / 26$ & $0.275^{\mathrm{NS}}$ \\
\hline $1.2 \mathrm{~B}+\mathrm{ve}$ & 104.14 & & & \\
\hline $1.3 \mathrm{O}+\mathrm{ve}$ & 101.75 & & & \\
\hline $1.4 \mathrm{AB}+\mathrm{ve}$ & 103.63 & & & \\
\hline \multicolumn{5}{|c|}{ 2) Days of pre-operative stay } \\
\hline $2.11-3$ & 103.13 & 1.045 & 28 & $0.305^{\mathrm{NS}}$ \\
\hline $2.24-6$ & 105.00 & & & \\
\hline \multicolumn{5}{|l|}{ Postoperative } \\
\hline \multicolumn{5}{|l|}{ 1) Haemoglobin } \\
\hline $1.13 \mathrm{gm} \%-6.9 \mathrm{gm} \%$ & 105.00 & 0.459 & $2 / 27$ & $0.637^{\mathrm{NS}}$ \\
\hline $1.27 \mathrm{gm} \%-11 \mathrm{gm} \%$ & 103.73 & & & \\
\hline $1.3>11 \mathrm{gm} \%$ & 101.00 & & & \\
\hline \multicolumn{5}{|l|}{ 2) Blood transfusion } \\
\hline 2.10 unit & 103.13 & 0.526 & $2 / 27$ & $0.597^{\mathrm{NS}}$ \\
\hline 2.21 unit & 105.00 & & & \\
\hline 2.32 unit & 105.00 & & & \\
\hline 2.43 unit & 72.00 & & & \\
\hline \multicolumn{5}{|c|}{ 3) Days of post-operative stay } \\
\hline $3.1 .6-8$ & 103.10 & 2.762 & 28 & $0.010^{*}$ \\
\hline $3.212-14$ & 111.00 & & & \\
\hline \multicolumn{5}{|c|}{ 4) Duration of hospital stay } \\
\hline $4.17-11$ & 102.91 & 1.729 & $2 / 27$ & $0.197^{\mathrm{NS}}$ \\
\hline $4.212-16$ & 105.50 & & & \\
\hline $4.317-21$ & 109.00 & & & \\
\hline \multicolumn{5}{|l|}{ 5)Day of discharge } \\
\hline $5.16^{\text {th }}$ day of operation & 103.11 & 2.827 & $3 / 26$ & $0.118^{\mathrm{NS}}$ \\
\hline $5.27^{\text {th }}$ day of operation & 101.85 & & & \\
\hline $5.38^{\text {th }}$ day of operation & 111.00 & & & \\
\hline $5.49^{\text {th }}$ day of operation & 103.63 & & & \\
\hline \multicolumn{5}{|c|}{ 6) Days of follow up after discharge } \\
\hline $6.17^{\text {th }}$ day & 102.80 & 0.435 & $5 / 24$ & $0.820^{\mathrm{NS}}$ \\
\hline $6.28^{\text {th }}$ day & 102.75 & & & \\
\hline $6.39^{\text {th }}$ day & 104.50 & & & \\
\hline $6.410^{\text {th }}$ day & 102.25 & & & \\
\hline $6.511^{\text {th }}$ day & 104.80 & & & \\
\hline $6.612^{\text {th }}$ day & 106.50 & & & \\
\hline
\end{tabular}




\section{DISCUSSION}

The present study shows that the types of operation were vaginal hysterectomy (35\%) and abdominal hysterectomy $(48.33 \%)$ in comparison and experimental group. The indication of vaginal hysterectomy was uterovaginal prolapse $(33.33 \%)$ whereas indication for abdominal hysterectomy were uterine fibroid (50\%) and AUB $(13.33 \%)$ in comparison and experimental group respectively which are consistent to the findings of Umeora et al., which shows that the type of operation were $33(52.2 \%)$ were abdominal hysterectomies while $29(46.8 \%)$ were vaginal hysterectomies. The indication of vaginal hysterectomy was uterovaginal hysterectomy and uterine fibroid was the commonest indication for abdominal hysterectomy. Teaching reduced emotional stress in experimental group which are similar to the findings Ozdemir et al., where significant difference in anxiety was recorded after implementation of training and relaxation exercises. The implementation of structure discharge planning was effective as indicating teaching enhanced ability scores in the area of physical activities, diet, emotional stress, psychological activities and sexual activities which are similar to the findings of Williams B shows that the importance of nurses providing specific and accurate verbal and printed discharge information to all patients was effective in the area of pain and wound management, activity, nutrition and complications generally. The structure discharge planning was effective as the women can able to perform self-care after return to home which are similar findings of Easton $\mathrm{K}$ et al shows that the beneficial effects on patient attitudes of the introduction of patient information leaflets, a preadmission clinic and a telephone advice service following discharge.

\section{Limitations}

Generalization of the findings is limited. In the present study, questionnaire regarding sexual activity were formed in present tense statement it can be further modified in future tense statement for better result.

\section{Recommendation}

The researcher recommended that the study need to be replicated on large sample to validate and generalize its findings. A descriptive study can be carried out to identify the barriers of self-care abilities of women underwent hysterectomy. A comparative study to assess the severity of depressive symptoms and poor self-care abilities among two different group of women underwent hysterectomy can be conducted.

\section{CONCLUSIONS}

The study was conducted to evaluate the effectiveness of structured discharge planning on self-care ability among women underwent hysterectomy in Maharishi Makandeshwar Institute of Medical Science \& Research,
Hospital, Mullana. The study concluded that the self-care ability in comparison group, most of the women had an average score but in experimental group, most of the women had very good score. The study finding reveal that there is effective of structured discharge planning where the women underwent hysterectomy can prevent the complication after hysterectomy.

\section{ACKNOWLEDGEMENT}

We express our appreciations to the respected officials of the institutes of M.M University Mullana, who cooperated with us for executing this research. The author would like to thank Dr. (Mrs) Jyoti Sarin and Mrs Poonam Sheoran for constant encouragement, detailed and constructive comments.

Funding: No funding sources

Conflict of interest: None declared

Ethical approval: The study was approved by the Institutional Ethics Committee

\section{REFERENCES}

1. Kazemi A, Homayuni M, Hassanzahraie R, Marufi M. Quality of life in physical and sexual dimensions among women understanding hysterectomy and hormone-therapy because of abnormal uterine bleeding. IJNMR. 2008;13(4):161-5.

2. World Health Organization. Constitution of the World Health Organization -Basic Documents, Forty-fifth edition, Supplement, October 2006.

3. Gun C, Komurcu N. The relationship between selfesteem and self-care agency in hysterectomy patients. Journal of Research in Nursing and Midwifery. 2013;2(7):89-95.

4. Hysterectomy available from: http://en.wikipedia.org/wiki/Hysterectomy.

5. Bharatnur S. Comparative Study Of Abdominal Versus Vaginal Hysterectomy In Non- Descent Cases. The Internet Journal of Gynecology and Obstetrics. 2010;15(2).

6. Vomvolaki E, Kalmantis K, Kioses E, Antsaklis A. The effect of hysterectomy on sexuality and psychological changes. The European Journal of Contraception and Reproductive Health Care. 2006;11(1):23-7.

7. McPherson K, Metcalfe M, Herbert A, Maresh M, Casbard A, Hargreaves J, et al. Severe complications of hysterectomy: the value study. BJOG: an international Journal of Obstetrics and Gynecology. 2004; 111:688-94.

8. Wong E, Yam C, Cheung A, Leung M, Chan F, Yeoh E. Barriers to effective discharge planning: a qualitative study investigating the perspectives of frontline healthcare professionals. BMC Health Services Research. 2011;11:242.

9. Bradford A, Meston C. Sexual Outcomes and Satisfaction with Hysterectomy: Influence of Patient 
Education. International Society for Sexual Medicine. 2007;4:106-14.

10. Ozdemir F, Pasinlioglu T. The effects of training and progressive Relaxation exercises on Anxiety Level after Hysterectomy. The New Journal of Medicine. 2009;26:102-7.

11. Williams B, supporting self-care of patients following general abdominal surgery, Journal of Clinical Nursing. 2008;17(5):584-92.
12. Easton K, Woodman NM. Influence of early discharge after discharge after hysterectomy on patient outcome and GP workloads. Journal of Obstetrics and Gynaecology. 2005;23(3):271-5.

Cite this article as: Nalini Devi K, Sheoran P, Sarin J. Effect of Structured Discharge Teaching after Hysterectomy. Int $\mathbf{J}$ Reprod Contracept Obstet Gynecol 2015;4:1384-93. 